Section Editor

John J. Millichap, MD

Teaching NeuroImages:

\title{
Acute isolated oculomotor nerve palsy of microvascular origin
}

\section{Figure Muscle edema in isolated oculomotor nerve palsy of microvascular origin}

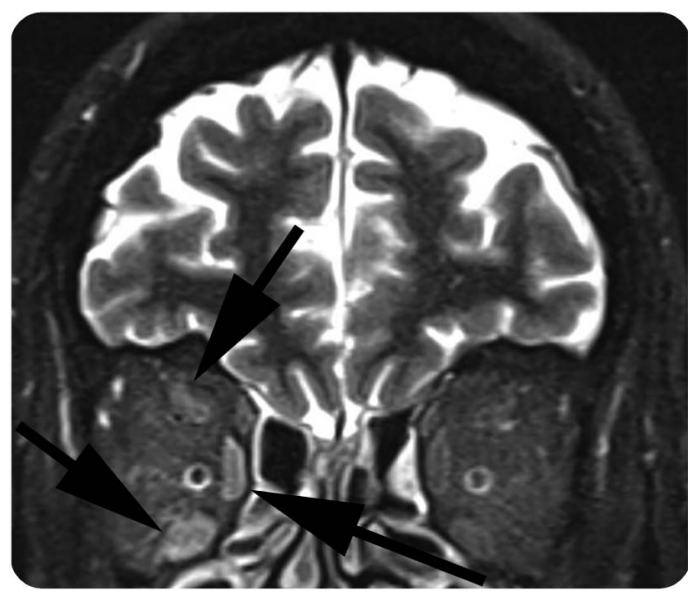

Coronal MRI, 10 days after acute symptom onset, shows increased signal intensity on T2-weighted imaging and increased volume of the muscles innervated by the right oculomotor nerve (arrows), suggesting muscle edema in the early subacute phase of denervation, which could be a potential imaging correlate of the clinically based diagnosis of microvascular oculomotor nerve palsy.

A 73-year-old man presented with acute-onset, right-sided ptosis and pain above the right eye. His medical history noted hypertension and diabetes. On physical examination, an isolated right oculomotor palsy was noted with preserved pupillary function. An MRI scan revealed isolated swelling of superior, inferior, and medial rectus muscle at the right side (figure). No cause other than presumed microvascular oculomotor nerve ischemia was shown. ${ }^{1}$ In patients aged 50 years or older, isolated ocular motor nerve palsies are most likely due to microvascular ischemia, but other etiologies such as aneurysm, brainstem stroke, and malignancy should be ruled out with MRI. ${ }^{1,2}$

\section{AUTHOR CONTRIBUTIONS}

Writing the manuscript: Dr. Ido van den Wijngaard. Review and discussion of the manuscript: Dr. Rogier Hagenbeek, Dr. Korné Jellema, Dr. Lycklama à Nijeholt.

\section{STUDY FUNDING}

No targeted funding reported.

\section{DISCLOSURE}

The authors report no disclosures relevant to the manuscript. Go to Neurology.org for full disclosures.

\section{REFERENCES}

1. Tamhankar MA, Biousse V, Ying GS, et al. Isolated third, fourth, and sixth cranial nerve palsies from presumed microvascular versus other causes: a prospective study. Ophthalmology 2013;120:2264-2269.

2. Kung NH, Van Stavern GP. Isolated ocular motor nerve palsies. Semin Neurol 2015;35:539-548.
Download teaching slides: Neurology.org
*These authors contributed equally to this work.

From the Departments of Neurology (I.R.v.d.W., K.J.) and Radiology (I.R.v.d.W., R.E.H., G.J.L.N.), Medical Center Haaglanden, the Hague, the Netherlands. 


\section{Neurology}

Teaching NeuroImages: Acute isolated oculomotor nerve palsy of microvascular origin Ido R. van den Wijngaard, Rogier E. Hagenbeek, Korné Jellema, et al.

Neurology 2016;87; 70

DOI 10.1212/WNL.0000000000002983

This information is current as of August 15, 2016

\begin{tabular}{|c|c|}
\hline $\begin{array}{l}\text { Updated Information \& } \\
\text { Services }\end{array}$ & $\begin{array}{l}\text { including high resolution figures, can be found at: } \\
\text { http://n.neurology.org/content/87/7/e70.full }\end{array}$ \\
\hline Supplementary Material & $\begin{array}{l}\text { Supplementary material can be found at: } \\
\text { http://n.neurology.org/content/suppl/2016/08/15/WNL.0000000000002 } \\
\text { 983.DC1 }\end{array}$ \\
\hline References & $\begin{array}{l}\text { This article cites } 2 \text { articles, } 0 \text { of which you can access for free at: } \\
\text { http://n.neurology.org/content/87/7/e70.full\#ref-list-1 }\end{array}$ \\
\hline Subspecialty Collections & $\begin{array}{l}\text { This article, along with others on similar topics, appears in the } \\
\text { following collection(s): } \\
\text { All Clinical Neurology } \\
\text { http://n.neurology.org/cgi/collection/all_clinical_neurology } \\
\text { MRI } \\
\text { http://n.neurology.org/cgi/collection/mri } \\
\text { Ocular motility } \\
\text { http://n.neurology.org/cgi/collection/ocular_motility } \\
\text { Other cerebrovascular disease/Stroke } \\
\text { http://n.neurology.org/cgi/collection/other_cerebrovascular_disease_s } \\
\text { troke }\end{array}$ \\
\hline Permissions \& Licensing & $\begin{array}{l}\text { Information about reproducing this article in parts (figures,tables) or in } \\
\text { its entirety can be found online at: } \\
\text { http://www.neurology.org/about/about_the_journal\#permissions }\end{array}$ \\
\hline Reprints & $\begin{array}{l}\text { Information about ordering reprints can be found online: } \\
\text { http://n.neurology.org/subscribers/advertise }\end{array}$ \\
\hline
\end{tabular}

Neurology ${ }^{\circledR}$ is the official journal of the American Academy of Neurology. Published continuously since 1951, it is now a weekly with 48 issues per year. Copyright @ 2016 American Academy of Neurology. All rights reserved. Print ISSN: 0028-3878. Online ISSN: 1526-632X.

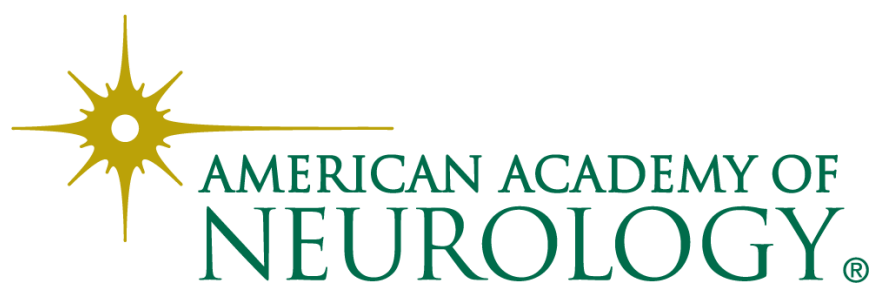

